\title{
Cellules solaires au GaAlAs/GaAs à faible et fort niveaux de courant. Optimisation détaillée du rendement de conversion
}

\author{
J. E. Bourée $(*)$ \\ Centre National d'Etudes Spatiales, 18, avenue Edouard-Belin, 31055 Toulouse Cedex, France \\ et F. Therez \\ Laboratoire d'Automatique et d'Analyse des Systèmes, C.N.R.S., \\ 7 , avenue du Colonel-Roche, 31400 Toulouse, France
}

(Reçu le 31 juillet 1979, révisé le 15 octobre 1979, accepté le 17 octobre 1979)

\begin{abstract}
Résumé. - L'étude proposée concerne l'optimisation du rendement des cellules au GaAlAs en fonction du niveau du courant, de la résistance série, de la température et en précise les limites dues aux différents paramètres physiques et technologiques. Une méthode détaillée de simulation numérique complète les résultats d'une étude classique des caractéristiques du fonctionnement des cellules solaires au GaAlAs/GaAs. L'analyse des principales propriétés photovoltaïques permet l'étude comparative des diverses structures. La théorie approfondie fait apparaître une modification des lois de conduction. Les valeurs des paramètres physiques qui rendent optimum le rendement de conversion à faible et fort niveaux de courant sont revues à partir de ces résultats. Les effets dus à l'élévation de la température, à la conduction non uniforme et aux distorsions des bandes d'énergie pour les dopages élevés sont développés.
\end{abstract}

\begin{abstract}
The efficiency of GaAlAs solar cells is optimized with respect to the concentration ratio, series resistance and temperature. The proposed study describes the limitations yielded by the various physical and technological parameters. A detailed numerical analysis extends the results of a classical one for the GaAlAs/GaAs solar cell characteristics. The analysis of the main photovoltaiic properties leads to compare some structures with the others. The advanced theory shows a conduction law modification. Then, the physical parameter values which yield the maximum conversion efficiency at low and high current levels are reviewed. The effects of a temperature increase, a non-uniform conduction and the distortions of the energy bands at high doping densities are reported.
\end{abstract}

1. Introduction. - Dans la recherche des rendements les plus élevés obtenus par la conversion photovoltaïque, les cellules solaires à l'arséniure de gallium s'avèrent les plus intéressantes. La largeur de la bande interdite du GaAs $\left(E_{\mathrm{g}}=1,425 \mathrm{eV}\right)$ est proche de la valeur nécessaire à la conversion la plus grande du rayonnement solaire. La puissance électrique de sortie sera délivrée sous une tension plus élevée que celle obtenue avec les autres matériaux et ces photopiles présenteront une meilleure résistance aux variations thermiques. Des rendements voisins de $22 \%$ sous un soleil (AM1) et $25 \%$ sous 178 soleils ont été mesurés dans deux laboratoires différents $[1,2]$.

D'autres cellules au GaAs ont fonctionné à des taux de concentration atteignant 1000 soleils et davantage [3]; quelques-unes dépassent même $19 \%$ de rendement sous 1700 soleils [4].

(*) Attaché de recherche au C.N.R.S.
La principale limitation des performances des cellules solaires au GaAs est la réduction du rendement de collecte provenant de la recombinaison élevée en surface, la vitesse de recombinaison variant de $10^{6}$ à $10^{7} \mathrm{~cm} / \mathrm{s}$. Plusieurs structures réduisant l'effet de la disparition des porteurs en surface ont été proposées :

1.1 StRUCTURE COMPORTANT UNE FENÊTRE (Fig. 1a) $[5,6]$. - Une mince couche ternaire $\mathrm{Ga}_{1-x} \mathrm{Al}_{x} \mathrm{As}$ à fort taux d'aluminium est épitaxiée sur l'homojonction $\mathrm{P}-\mathrm{N}$ en GaAs. Les photons d'énergie inférieure à la largeur de bande interdite du GaAlAs sont absorbés uniquement dans la couche GaAs et les porteurs ainsi générés par le rayonnement solaire sont d'autant plus collectés à la jonction que les états d'interface à l'hétérojonction présentent une densité faible

$$
\left(S_{1} \simeq 10^{4} \mathrm{~cm} / \mathrm{s}\right) \text {. }
$$




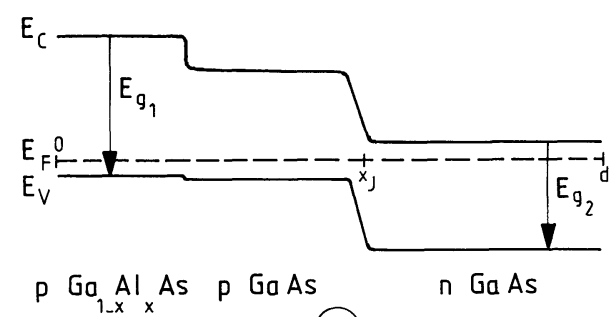

(a)

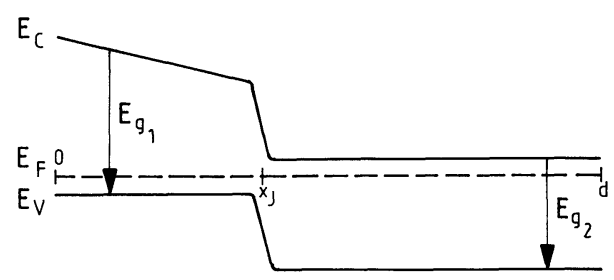

$P \mathrm{Ga}_{1_{-} \mathrm{x}} \mathrm{Al}_{\mathrm{x}} \mathrm{As}$

$n$ Ga As

(b)

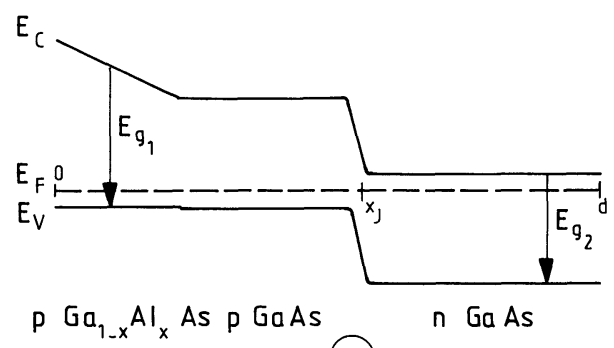

(C)

Fig. 1. - Schémas de bandes des cellules étudiées : Cellule (a) : structure $\mathrm{PGaAlAs} / \mathrm{PGaAs} / \mathrm{NGaAs}$ à hétérojonction ou à couche fenêtre. Cellule (b) : structure $\mathrm{PGaAlAs} / \mathrm{NGaAs}$ à gradient de bande interdite. Cellule (c) : structure $\mathrm{PGaAlAs} / \mathrm{PGaAs} / \mathrm{NGaAs}$ à gradient de bande interdite avec couche intermédiaire.

[Energy band diagrams of the three selected cells : Cell (a) : heteroface or window PGaAlAs/PGaAs/NGaAs structure. Cell (b) : graded bandgap PGaAlAs/NGaAs structure. Cell (c) : graded bandgap $\mathrm{PGaAlAs} / \mathrm{PGaAs} / \mathrm{NGaAs}$ structure.]

1.2 HOMOJONCTION DONT LA COUCHE SUPERFICIELLE EST AMINCIE [7]. — En rôdant suffisamment la couche avant jusqu'à l'épaisseur de quelques centaines d'angströms, les porteurs en excès sont générés pratiquement dans la région de base et la collecte est peu influencée par la recombinaison en surface. Un champ électrique face arrière dû à un profil de dopage permet de réduire le courant d'obscurité et d'obtenir une tension en circuit ouvert plus grande.

1.3 STRUCTURE COMPORTANT UN CHAMP D'ENTRAîNEMENT [1]. - La réalisation d'un gradient de dopage ou de bande interdite (Fig. $1 b$ ) produit un champ électrique interne qui entraîne les porteurs minoritaires vers la jonction. L'effet de la recombinaison en surface est réduit et le courant de court-circuit devient plus grand.

L'étude proposée concerne l'optimisation du rendement des cellules au GaAlAs/GaAs en fonction du taux de concentration, de la résistance série, de la température et en précise les limites dues aux différents paramètres physiques et technologiques. Un modèle simplifié donne une évaluation des caractéristiques des structures traditionnelles et en particulier celles dont les schémas de bandes sont représentés sur les figures $1 a$ et $1 b$. Les résultats classiques sont complétés par une simulation numérique approfondie. L'étude prenant en compte les phénomènes de recombinaison dans la charge d'espace apporte une modification significative aux grandeurs photovoltaïques déduites du modèle simplifié. Le programme détaillé optimise essentiellement la structure de la figure $1 c$.

L'évolution des principaux facteurs photovoltaïques est considérée aux forts éclairements. Les mécanismes physiques liés à l'échauffement de la cellule, à la répartition inhomogène des densités de courant dans la région épitaxiée et les perturbations dues aux dopages élevés sont analysés.

2. Etude comparative des diverses structures. Modèle simplifié. - Une analyse approchée des structures dont les schémas de bandes sont représentés sur la figure 1 permet d'obtenir leurs principales propriétés photovoltaïques dans le cadre des hypothèses suivantes :

- le coefficient d'absorption du GaAs et des composés GaAlAs varie comme la racine carrée de l'énergie mesurée à partir du ou des seuils d'absorption correspondants,

- la composante du champ électrique interne produite par la variation de l'affinité électronique n'est pas considérée,

- les phénomènes de génération-recombinaison ne sont pas pris en compte dans la zone de charge d'espace.

Les équations fondamentales des semiconducteurs décrivant le comportement des porteurs dans les régions $\mathrm{P}$ et $\mathrm{N}$ sont les lois de transport :

$$
\begin{aligned}
& J_{\mathrm{N}}=q D_{\mathrm{N}} N \frac{\mathrm{d} \varphi_{\mathrm{N}}}{\mathrm{d} x}=q D_{\mathrm{N}} \frac{\mathrm{d} N}{\mathrm{~d} x}+q \mu_{\mathrm{N}} N E \\
& J_{\mathrm{P}}=-q D_{\mathrm{P}} P \frac{\mathrm{d} \varphi_{\mathrm{P}}}{\mathrm{d} x}=-q D_{\mathrm{P}} \frac{\mathrm{d} P}{\mathrm{~d} x}+q \mu_{\mathrm{P}} P E
\end{aligned}
$$

et les équations de continuité en régime permanent :

$$
\begin{array}{r}
\frac{1}{q} \frac{\mathrm{d} J_{\mathrm{N}}}{\mathrm{d} x}-U+G=0 \\
-\frac{1}{q} \frac{\mathrm{d} J_{\mathrm{P}}}{\mathrm{d} x}-U+G=0
\end{array}
$$

dans lesquelles $J_{\mathrm{N}}$ et $J_{\mathrm{P}}$ représentent les densités de courant des électrons et des trous, $\varphi_{\mathrm{N}}, \varphi_{\mathrm{P}}, U$ et $G$ désignant respectivement les potentiels de Fermi normalisés ainsi que les taux de recombinaison et de génération des porteurs. 
$D_{\mathrm{N}}, \mu_{\mathrm{N}}$ et $D_{\mathrm{P}}, \mu_{\mathrm{P}}$ représentent successivement le coefficient de diffusion et la mobilité des électrons et des trous.

$E$ est le champ électrique d'entraînement associé à un gradient de dopage ou de bande interdite. Le champ électrique dans la couche (épitaxiée) de type $\mathbf{P}$ est uniforme, celui associé à la couche de base est supposé nul.

Dans les conditions de l'injection faible $\left(N_{\mathrm{P}} \ll P_{0}\right.$ et $P_{\mathrm{N}} \ll N_{0}$ ), les taux de recombinaison $U_{\mathrm{N}}$ et $U_{\mathrm{P}}$ s'expriment dans les régions $P$ et $N$ en fonction des porteurs minoritaires en excès :

$$
U_{\mathrm{N}}=\frac{N-N_{0}}{\tau_{\mathrm{N}}}, \quad U_{\mathrm{P}}=\frac{P-P_{0}}{\tau_{\mathrm{P}}}
$$

$\tau_{\mathbf{N}}$ et $\tau_{\mathbf{P}}$ désignant les durées de vie des électrons et des trous.

Le taux de génération à une longueur d'onde donnée $G(x, \lambda)$ se déduit du flux de photons $I(x, \lambda)$ et du coefficient d'absorption $\alpha(x, \lambda)$ en négligeant les pertes par réflexion : avec

$$
G(x, \lambda) \mathrm{d} x=-\mathrm{d} I(x, \lambda)=\alpha(x, \lambda) I(x, \lambda) \mathrm{d} x
$$

$$
\alpha(x, \lambda)=A\left(\frac{h c}{\lambda}-E_{\mathrm{g}}-q E x\right)^{1 / 2}
$$

$A$ est une constante d'absorption relative au matériau GaAs.

Le calcul des densités de courant de lumière nécessite la détermination des solutions des éqs. (1) à (4). Ces solutions doivent satisfaire les conditions aux limites imposées aux extrémités de la cellule :

- sur la face arrière de la cellule, en $x=d$, le contact est supposé ohmique. Ainsi, pour chaque longueur d'onde :

$$
\Delta P(d)=P(d)-P_{0}=0
$$

- sur la surface illuminée, en $x=0$, l'équation de continuité des courants s'écrit pour chaque longueur d'onde :

$$
J_{\mathrm{N}}(0)=q S\left(N(0)-N_{0}\right)
$$

$S$ étant la vitesse de recombinaison en surface.

Le rendement de collecte $\gamma_{N}(\lambda)$ (ou $\left.\gamma_{P}(\lambda)\right)$ dans la région $P$ (ou $N$ ) se définit comme le rapport du nombre des électrons de la région $P$ (ou des trous de la région $\mathrm{N}$ ) collectés par la jonction au nombre de photons incidents pour une longueur d'onde déterminée. Si $I_{0}(\lambda), J_{\mathrm{LN}}(\lambda)$ et $J_{\mathrm{LP}}(\lambda)$ représentent respectivement, à $\lambda$ donné, le flux de photons incidents et les densités de courant de lumière dues aux électrons et aux trous minoritaires, le rendement de collecte total $\gamma(\lambda)$ s'écrit :

$$
\gamma(\lambda)=\gamma_{\mathbf{N}}(\lambda)+\gamma_{\mathbf{P}}(\lambda)=\frac{J_{\mathbf{L N}}(\lambda)+J_{\mathrm{LP}}(\lambda)}{q I_{0}(\lambda)}
$$

Les densités de courant total traversant la jonction, en $x=x_{\mathrm{J}}$, se mettent donc sous la forme :

$$
\begin{aligned}
& J_{\mathrm{N}}\left(x_{\mathrm{J}}\right)=-\int_{0}^{\lambda_{E_{\mathrm{g}}}} j_{\mathrm{LN}}(\lambda) \mathrm{d} \lambda+J_{\mathrm{SN}}\left[\exp \left(\frac{q V_{\mathrm{J}}}{k T}\right)-1\right] \\
& J_{\mathrm{P}}\left(x_{\mathrm{J}}\right)=-\int_{0}^{\lambda_{E_{\mathrm{g}}}} j_{\mathrm{LP}}(\lambda) \mathrm{d} \lambda+J_{\mathrm{SP}}\left[\exp \left(\frac{q V_{\mathrm{J}}}{k T}\right)-1\right]
\end{aligned}
$$

expressions dans lesquelles $J_{\mathrm{SN}}$ et $J_{\mathrm{SP}}$ désignent les densités de courant de saturation dues aux électrons et aux trous, calculées dans le cadre du modèle de diffusion.

Les densités des porteurs à la jonction, en $x=x_{\mathrm{J}}$, suivent la loi exponentielle de Boltzmann en fonction de la tension $V_{\mathrm{J}}$. En l'absence de tension $\left(V_{\mathrm{J}}=0\right)$, le champ interne de la jonction annule l'excès des porteurs générés en son plan. La différence de potentiel $V_{\mathrm{J}}$ à la jonction se déduit de la tension $V$ aux bornes de la cellule suivant la relation :

$$
V_{\mathrm{J}}=V-R_{\mathrm{S}}\left(J_{\mathrm{N}}+J_{\mathbf{P}}\right)
$$

dans laquelle $R_{\mathrm{S}}$, la résistance série spécifique exprimée en $\Omega . \mathrm{cm}^{2}$, est définie comme l'inverse de la conductance par unité de surface. Désormais, la surface de la cellule sera prise égale à l'unité $\left(1 \mathrm{~cm}^{2}\right)$ et la résistance série sera exprimée en $\Omega$.

Tous les résultats présentés dans la suite ont été obtenus en adoptant le spectre solaire hors atmosphère AMO déterminé par Thekaekara [8] (le rayonnement solaire total correspondant est de $135,3 \mathrm{~mW} . \mathrm{cm}^{-2}$ ).

Ce modèle a été appliqué tout d'abord à l'étude des deux homostructures $\mathrm{P}^{+} / \mathrm{N}$ et $\mathrm{N}^{+} / \mathbf{P}$ à face avant amincie. Le tableau comparatif 1 indique, pour une résistance série nulle, les valeurs des rendements obtenus en faisant varier l'épaisseur de la couche superficielle pour différentes vitesses de recombinaison en surface. Nous remarquons qu'en diminuant l'épaisseur $x_{\mathrm{J}}$, le rendement passe par un maximum qui se déplace vers les faibles valeurs de $x_{\mathrm{J}}$ au fur et à mesure de l'accroissement de la vitesse de recombinaison en surface. Ceci s'explique par les effets combinés de l'absorption, de la vitesse de recombinaison en surface, de l'épaisseur et de la longueur de diffusion de la couche superficielle. Par exemple, les longueurs de diffusion associées aux couches superficielles des deux structures $\mathrm{P}^{+} \mathrm{N}$ et $\mathrm{N}^{+} \mathrm{P}$ sont respectivement

$$
L_{\mathrm{N}}=2,2 \mu \mathrm{m} \quad \text { et } \quad L_{\mathrm{P}}=1 \mu \mathrm{m} .
$$

Le calcul montre, à vitesse de recombinaison égale pour ces structures et quelle qu'en soit sa valeur, que la première atteint son maximum de rendement pour une profondeur de la région $\mathrm{P}^{+}$qui est double de la profondeur de la région $\mathrm{N}^{+}$rendant maximum le rendement de la seconde. Notons que la structure 
Tableau I. - Rendement AMO $(\%)$ calculé en fonction de l'épaisseur de la face avant $x_{\mathrm{J}}$ pour différentes valeurs de la vitesse de recombinaison en surface $\left(R_{\mathrm{S}}=0\right)$.

\begin{tabular}{|c|c|c|c|c|}
\hline \multicolumn{5}{|c|}{ Structure $\mathrm{P}^{+} \mathrm{GaAs} / \mathrm{NGaAs}$} \\
\hline \multirow{2}{*}{\multicolumn{2}{|c|}{$\begin{aligned} E_{\mathrm{g}_{1}} & =E_{\mathrm{g} 2}=1,425 \mathrm{eV} \\
L_{\mathrm{N}} & =2,2 \mu \mathrm{m}\left(N_{\mathrm{A}}=5 \times 10^{18} \mathrm{~cm}^{-3}\right) \\
\mu_{\mathrm{N}} & =3000 \mathrm{~cm}^{2} \cdot \mathrm{V}^{-1} \cdot \mathrm{s}^{-1}\end{aligned}$}} & \multirow{2}{*}{\multicolumn{3}{|c|}{$\begin{array}{l}d-x_{\mathrm{J}}=4 \mu \mathrm{m} \\
L_{\mathrm{P}}=3,5 \mu \mathrm{m}\left(N_{\mathrm{D}}=10^{17} \mathrm{~cm}^{-3}\right) \\
\mu_{\mathrm{P}}=400 \mathrm{~cm}^{2} \cdot \mathrm{V}^{-1} \cdot \mathrm{s}^{-1}\end{array}$}} \\
\hline & & & & \\
\hline$S(\mathrm{~cm} / \mathrm{s})$ & 0 & $10^{5}$ & $10^{\prime \prime}$ & $10^{7}$ \\
\hline 2 & 16,2 & 14,1 & 8 & 4,7 \\
\hline 1 & 20,6 & 19,2 & 13,4 & 8,5 \\
\hline 0,5 & 21,3 & 20,7 & 17,3 & 12,5 \\
\hline 0,25 & $\overline{20,9}$ & 20,7 & 19,2 & 15,7 \\
\hline 0,1 & 20,2 & $\overline{20,2}$ & 19,7 & 17,9 \\
\hline 0,05 & 19,9 & 19,9 & $\overline{19,6}$ & 18,5 \\
\hline
\end{tabular}

\section{Structure $\mathrm{N}^{+} \mathrm{GaAs} / \mathrm{PGaAs}$}

$$
\begin{array}{ll}
E_{\mathrm{g}_{1}}=E_{\mathrm{g} 2}=1,425 \mathrm{eV} & d-x_{\mathrm{J}}=4 \mu \mathrm{m} \\
L_{\mathrm{P}}=1 \mu \mathrm{m}\left(N_{\mathrm{D}}=5 \times 10^{18} \mathrm{~cm}^{-3}\right) & L_{\mathrm{N}}=7 \mu \mathrm{m}\left(N_{\mathrm{A}}=10^{17} \mathrm{~cm}^{-3}\right) \\
\mu_{\mathrm{P}}=400 \mathrm{~cm}^{2} \cdot \mathrm{V}^{-1} \cdot \mathrm{s}^{-1} & \mu_{\mathrm{N}}=3000 \mathrm{~cm}^{2} \cdot \mathrm{V}^{-1} \cdot \mathrm{s}^{-1}
\end{array}
$$

\begin{tabular}{l|rrrr}
\hline$S(\mathrm{~cm} / \mathrm{s})$ & & & & \\
$\lambda, \mu \mathrm{m})$ & 0 & $10^{5}$ & $10^{\prime \prime}$ & $10^{7}$ \\
\hline 2 & 7,3 & 5,7 & 4,3 & 4,1 \\
1 & 15,4 & 11,9 & 8,3 & 7,5 \\
0,5 & 19,3 & 16,7 & 12,5 & 11,2 \\
0,25 & $\underline{19,9}$ & 18,8 & 15,8 & 14,4 \\
0,1 & 19,5 & $\underline{19,3}$ & 18,2 & 17 \\
0,05 & 19,3 & 19,3 & $\underline{18,8}$ & $\underline{18,1}$
\end{tabular}

$\mathrm{P}^{+} \mathrm{N}$ est mieux adaptée à la profondeur effective $(\sim 1 \mu \mathrm{m})$ de l'absorption du rayonnement.

Le modèle est ensuite utilisé pour comparer une cellule ayant un gradient de bande interdite dans la face avant [9] (Fig. 1b) à la cellule à homojonction correspondante. La première cellule a les paramètres suivants :

$$
\begin{aligned}
E_{\mathrm{g}_{1}} & =1,84 \mathrm{eV}, & E_{\mathrm{g}_{2}} & =1,425 \mathrm{eV}, \\
x_{\mathrm{J}} & =1,3 \mu \mathrm{m}, & d & =16,3 \mu \mathrm{m}, \\
N_{\mathrm{A}} & =2 \cdot\left(10^{18}\right) \mathrm{cm}^{-3}, & N_{\mathrm{D}} & =10^{17} \mathrm{~cm}^{-3}, \\
\mu_{\mathrm{N}} & =2500 \mathrm{~cm}^{2} \cdot \mathrm{V}^{-1} \cdot \mathrm{s}^{-1}, & \mu_{\mathrm{P}} & =150 \mathrm{~cm}^{2} \cdot \mathrm{V}^{-1} \cdot \mathrm{s}^{-1}, \\
L_{\mathrm{N}} & =3 \mu \mathrm{m}, & L_{\mathrm{P}} & =2 \mu \mathrm{m} .
\end{aligned}
$$

La deuxième cellule a les mêmes paramètres à l'exception de $E_{\mathrm{g}_{1}}=1,425 \mathrm{eV}$. La figure 2 montre le rendement de collecte de ces deux structures en fonction de la longueur d'onde pour plusieurs valeurs de la vitesse de recombinaison en surface : la collecte est nettement améliorée aux courtes longueurs d'onde dans le cas de la structure $1 b$ car le champ interne de $3192 \mathrm{~V} / \mathrm{cm}$ entraîne fortement les électrons minoritaires de la couche $P$ vers la jonction. Pour

$$
S=5 \times 10^{5} \mathrm{~cm} / \mathrm{s},
$$

le rendement calculé pour la cellule type $1 b$, soit $23,2 \%$, est nettement supérieur à celui de l'homojonction : $14,7 \%$.

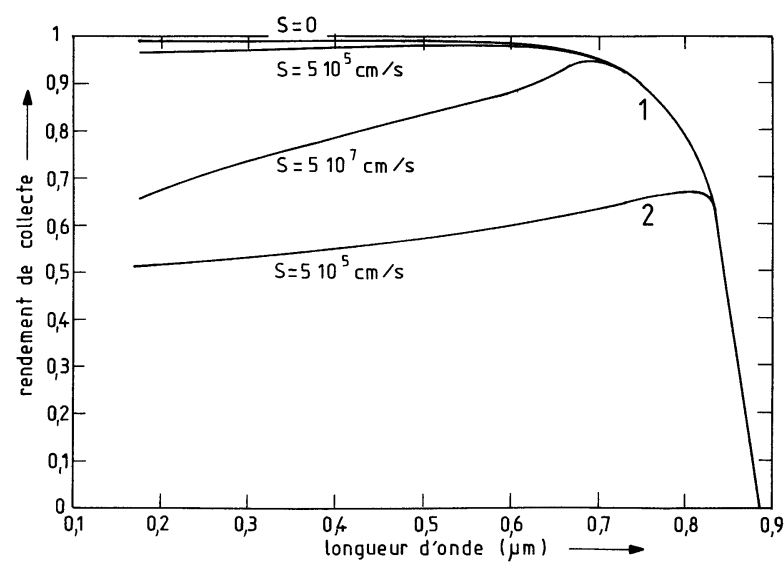

Fig. 2. - Variation du rendement de collecte interne en fonction de la longueur d'onde calculée : $1:$ pour la cellule (b) $; 2$ : pour la cellule à homojonction; pour plusieurs valeurs de la vitesse de recombinaison en surface $S$.

[Computed internal collection efficiency versus wavelength : 1 : for cell (b) and 2 : for homostructure cell, for several surface recombination velocity values.]

Enfin la structure type $1 b$, définie précédemment, est mise en balance avec la structure type $1 c$ équivalente. Pour cette dernière, le seul paramètre nouveau introduit est l'épaisseur de la couche $\mathbf{P}$ intermédiaire, la mobilité et la longueur de diffusion associées étant prises identiques à celles de la couche $P$ superficielle. Le calcul montre que le rendement de collecte de la cellule $1 c$ est renforcé aux grandes longueurs d'onde car les photons d'énergie voisine de $1,425 \mathrm{eV}$ sont absorbés dans la couche intermédiaire pour laquelle $L_{\mathrm{N}}$ est supérieure à $L_{\mathrm{P}}$; au contraire son rendement de collecte est diminué aux courtes longueurs d'onde car, les photons énergétiques étant absorbés plus loin de la jonction, les porteurs créés ont tendance à se recombiner dans la région intermédiaire. L'épaisseur optimum permettant d'atteindre le rendement de conversion maximum pour $S=5 \times 10^{5} \mathrm{~cm} / \mathrm{s}$ est trouvée voisine de $1 \mu \mathrm{m}$.

En conclusion le modèle proposé ici est simple dans son emploi et permet rapidement de choisir, parmi plusieurs structures mises en compétition, celle qui aura le meilleur rendement. En contrepartie il surestime les valeurs des rendements, notamment en ne prenant pas en compte la génération-recombinaison dans la région de charge d'espace.

3. Simulation numérique détaillée. - Un programme détaillé est utilisé pour résoudre les équations fondamentales des semiconducteurs. Les phénomènes de transport sont régis par les relations (1), (2) et l'équation de Poisson suivante :

$$
\begin{aligned}
\frac{\mathrm{d}^{2} \varphi}{\mathrm{d} x^{2}}=-\frac{q}{U_{\mathrm{T}} \varepsilon}\left(P-N+N_{\mathrm{D}}-\right. & \left.N_{\mathrm{A}}\right)- \\
& -\frac{1}{U_{\mathrm{T}} \varepsilon} \frac{\mathrm{d} \varphi}{\mathrm{d} x} \frac{\mathrm{d} \varepsilon}{\mathrm{d} x}
\end{aligned}
$$


où $\varphi=V / U_{\mathrm{T}}$ est le potentiel normalisé et $U_{\mathrm{T}}=k T / q$ est le potentiel thermodynamique.

Le gradient de bande est formulé par la variation des densités des porteurs $N$ et $P$ en fonction de la densité $n_{\mathrm{i}}\left(x_{\mathrm{J}}\right)$ de référence. La structure de la figure $1 c$ est retenue pour les simulations numériques

$$
\begin{aligned}
& N(x)=n_{\mathrm{i}}\left(x_{\mathrm{J}}\right) \cdot \exp \left(\varphi_{\mathrm{N}}+\varphi\right) \cdot \exp \left(\varphi_{\theta \mathrm{N}}\right) \\
& P(x)=n_{\mathrm{i}}\left(x_{\mathrm{J}}\right) \cdot \exp \left(\varphi_{\mathrm{P}}-\varphi\right) \cdot \exp \left(-\varphi_{\theta \mathrm{P}}\right)
\end{aligned}
$$

$n_{\mathrm{i}}\left(x_{\mathrm{J}}\right)$ est la concentration intrinsèque du matériau GaAs. L'abscisse $x_{\mathrm{J}}$ est la distance qui sépare la surface éclairée du point de l'homojonction où le niveau de Fermi intrinsèque croise le niveau de Fermi. Le zéro des potentiels est choisi à la distance $x_{\mathrm{J}}$. Les quantités $\varphi_{\theta \mathrm{N}}$ et $\varphi_{\theta \mathrm{P}}$ sont les paramètres de bande et dépendent uniquement de la distance par l'intermédiaire de $E_{\mathrm{g}}(x), N_{\mathrm{c}}(x), N_{\mathrm{v}}(x)$ et de l'affinité électronique $\chi(x)$

$$
\begin{array}{r}
\varphi_{\theta \mathrm{N}}=\ln \frac{n_{\mathrm{i}}(x)}{n_{\mathrm{i}}\left(x_{\mathrm{J}}\right)}+\frac{\chi(x)-\chi\left(x_{\mathrm{J}}\right)}{k T}+\frac{E_{\mathrm{g}}(x)-E_{\mathrm{g}}\left(x_{\mathrm{J}}\right)}{2 k T}+ \\
+\frac{1}{2} \ln \frac{N_{\mathrm{c}}(x) \cdot N_{\mathrm{v}}\left(x_{\mathrm{J}}\right)}{N_{\mathrm{v}}(x) \cdot N_{\mathrm{c}}\left(x_{\mathrm{J}}\right)}
\end{array}
$$

et

$$
\varphi_{\theta \mathrm{P}}=\varphi_{\theta \mathrm{N}}-2 \ln \frac{n_{\mathrm{i}}(x)}{n_{\mathrm{i}}\left(x_{\mathrm{J}}\right)}
$$

L'ensemble du système des éqs. (3), (4), (14) et les relations de liaison (15) et (16) est similaire aux lois de transport dans une homojonction aux variables $\varphi_{\theta \mathrm{N}}$ et $\varphi_{\theta \mathrm{P}}$ près d'une part et à la formulation de la loi de Poisson d'autre part. Cependant ces différences ne faisant intervenir que des quantités fonction de la position $x$, n'entraînent que des modifications de formulation et ne remettent pas en cause les méthodes numériques de résolution [10].

Nous définissons les variables intermédiaires suivantes :

$$
U_{\mathrm{N}}=\exp \varphi_{\mathrm{N}} \quad \text { et } \quad U_{\mathrm{P}}=\exp \varphi_{\mathrm{P}}
$$

et les facteurs de normalisation :

$$
\begin{array}{ll}
L_{\mathrm{D}}^{2}=\frac{\varepsilon_{1} \cdot k T}{q^{2} n_{\mathrm{i}}\left(x_{\mathrm{J}}\right)} ; & X=\frac{x}{L_{\mathrm{D}}} ; \\
\varepsilon_{\mathrm{PS}}=\frac{\varepsilon_{\mathrm{i}}}{\varepsilon_{1}} ; & D O P=\frac{N_{\mathrm{D}}-N_{\mathrm{A}}}{n_{\mathrm{i}}\left(x_{\mathrm{J}}\right)}
\end{array}
$$

où

- $L_{\mathrm{D}}$ est la longueur de Debye,

- $\varepsilon_{1}$ la constante diélectrique du matériau homogène de GaAs.

Dans ces conditions le système à résoudre s'écrit :

$$
\left.\begin{array}{r}
\frac{\mathrm{d}}{\mathrm{d} X}\left[D_{\mathrm{N}} \cdot \exp (\varphi) \cdot \exp \left(\varphi_{\theta \mathrm{N}}\right) \cdot \frac{\left.\left.\mathrm{d} U_{\mathrm{N}}\right]-\frac{U-G}{\mathrm{~d} X}\right]-L_{\mathrm{D}}^{2}=0}{n_{\mathrm{i}}\left(x_{\mathrm{J}}\right)}=0\right. \\
\frac{\mathrm{d}}{\mathrm{d} X}\left[D_{\mathrm{P}} \cdot \exp (-\varphi) \cdot \exp \left(-\varphi_{\theta \mathrm{P}}\right) \cdot \frac{\left.\left.\mathrm{d} U_{\mathrm{P}}\right]-\frac{U-G}{\mathrm{~d} X}\right]-L_{\mathrm{D}}^{2}=0}{n_{\mathrm{i}}\left(x_{\mathrm{J}}\right)}\right. \\
\varepsilon_{\mathrm{PS}} \cdot \frac{\mathrm{d}^{2} \varphi}{\mathrm{d} X^{2}}-U_{\mathrm{N}} \cdot \exp (\varphi) \cdot \exp \left(\varphi_{\theta \mathrm{N}}\right)+U_{\mathrm{P}} \cdot \exp (-\varphi) \cdot \exp \left(-\varphi_{\theta \mathrm{P}}\right)+D O P+\frac{\mathrm{d} \varepsilon_{\mathrm{PS}}}{\mathrm{d} X} \cdot \frac{\mathrm{d} \varphi}{\mathrm{d} X}=0
\end{array}\right\}
$$

La discrétisation des trois équations précédentes en différences finies permet d'exprimer les variables $\varphi$, $\varphi_{\theta \mathrm{N}}, \varphi_{\theta \mathrm{P}}$ et $U_{\mathrm{N}}, U_{\mathrm{P}}$ au point $\mathrm{i}$ en fonction des valeurs aux points $\mathrm{i}-1$ et $\mathrm{i}+1$ du maillage décrivant le composant.

Les grandeurs $\varphi, N$ et $P$ entre deux itérations $l-1$ et $l$ sont reliées aux variables indépendantes $\delta \varphi_{\mathrm{i}}$, $\delta N_{\mathrm{i}}$ et $\delta P_{\mathrm{i}}$ suivant les expressions :

$$
\left.\begin{array}{rl}
\varphi_{\mathrm{i}}^{l} & =\varphi_{\mathrm{i}}^{l-1}+\delta \varphi_{\mathrm{i}}^{l} \\
N_{\mathrm{i}}^{l} & =N_{\mathrm{i}}^{l-1} \cdot\left(1+\delta \varphi_{\mathrm{i}}^{l}\right)+\delta N_{\mathrm{i}}^{l} \\
P_{\mathrm{i}}^{l} & =P_{\mathrm{i}}^{l-1} \cdot\left(1-\delta \varphi_{\mathrm{i}}^{l}\right)+\delta P_{\mathrm{i}}^{l} \cdot
\end{array}\right\}
$$

Le programme calcule les accroissements $\delta \varphi_{\mathrm{i}}^{l}$, $\delta N_{\mathrm{i}}^{l}$ et $\delta P_{\mathrm{i}}^{l}$ qu'il faut ajouter à la solution de l'itération $l-1$ pour obtenir la solution de l'itération $l$. L'initialisation est effectuée à partir d'une répartition approchée du potentiel et des densités des porteurs aux extrémités du composant. La convergence vers la solution précise nécessite moins de 10 itérations; l'écart $\delta \varphi_{\mathrm{i}}^{l} / \varphi_{\mathrm{i}}^{l}$ est alors inférieur à $10^{-7}$.
Le taux de génération optique est identique à la formulation développée dans le paragraphe précédent (éq. (6)).

Les mécanismes électroniques négligés dans les modèles de premier ordre, tels que la contribution des taux de génération-recombinaison dans la région de charge d'espace et la variation de la durée de vie en fonction d'une répartition non uniforme des atomes d'impureté ou des centres de recombinaison peuvent être inclus dans le schéma numérique.

Le processus pris en compte dans le programme numérique est le taux de recombinaison-piégeage délocalisé de Shockley, Read et Hall :

$$
U=\frac{N \cdot P-n_{\mathrm{i}}^{2}}{\tau_{\mathrm{P}}(x) \cdot\left[N+N_{1}\right]+\tau_{\mathrm{N}}(x) \cdot\left[P+P_{1}\right]} .
$$

Les densités $N_{1}$ et $P_{1}$ sont respectivement le nombre des électrons et des trous lorsque le niveau de Fermi est confondu avec le niveau piège $E_{\mathrm{T}}$.

Les conditions aux limites sont données par la 
relation (8) pour le contact face arrière. Les densités des porteurs libres de la face éclairée résultent de l'excitation optique, des phénomènes d'injection et de la valeur de la vitesse de recombinaison en surface. La détermination des densités des porteurs $N(0)$ et $P(0)$ en surface est effectuée par des estimations successives. Les densités de courant sont déduites à partir d'une valeur approchée de $N(0)$ et $P(0)$; ces courants nous donnent la nouvelle concentration des porteurs qui sera alors utilisée comme initialisation. La convergence vers une valeur constante est rapidement obtenue car l'influence du changement de la densité des porteurs en surface est prise en compte par la modification du taux de génération-recombinaison, $U-G$ [11]. La validité du résultat est vérifiée par la relation (9).

Les caractéristiques courant-tension de la cellule solaire dont le schéma de bandes est identique à celui de la figure $1 c$, sont simulées. Les valeurs des densités des atomes d'impureté, des durées de vie et des longueurs de diffusion des porteurs minoritaires dans les diverses régions de la structure sont fixées comme suit :

$-N_{\mathrm{D}}=10^{17} \mathrm{~cm}^{-3}, \tau_{\mathrm{P}}=10,3 \mathrm{~ns}$ et $L_{\mathrm{P}}=2 \mu \mathrm{m}$ pour le GaAs de type $\mathrm{N}$,

$-N_{\mathrm{A}}=2 .\left(10^{18}\right) \mathrm{cm}^{-3}, \tau_{\mathrm{N}}=1,4 \mathrm{~ns}$ et $L_{\mathrm{N}}=3 \mu \mathrm{m}$ pour le GaAs de type $\mathrm{P}$,

- la profondeur de la région GaAlAs est égale à $0,25 \mu \mathrm{m}$, la largeur de la bande interdite en surface atteint la valeur de $1,84 \mathrm{eV}$.

Dans ces conditions, la figure 3 montre successivement la courbe 1 de la caractéristique à l'obscurité $(G=0)$, la courbe 2 de la variation du courant $-J_{\mathrm{SC}}+J_{\text {total }}$ de la cellule éclairée et dont le taux de génération est calculé à partir du spectre AMO [8], et la courbe 3 de la loi de conduction de la diode déduite d'un modèle simple de diffusion. La caractéristique 2 rendant compte de la loi de conduction de la cellule et de ses performances photovoltaiqques n'est pas linéaire à la différence du résultat du modèle de premier ordre, rappelé sur la courbe 3.

Nous observons que la caractéristique $J(V)$ sous éclairement n'est pas identifiée par la superposition de la caractéristique à l'obscurité et de celle du générateur de courant de valeur $J_{\mathrm{SC}}$. Le détail du calcul montre que les phénomènes de génération-recombinaison, essentiellement non linéaires dans la région de transition de l'homojonction, contribuent à la différence entre les courbes 1 et 2 . En particulier, l'excès des porteurs minoritaires aux limites de la charge d'espace, pour la condition de court-circuit de la cellule, ne s'annule pas. A l'intérieur de la région dépeuplée les densités des porteurs diminuent jusqu'à une valeur qui reste supérieure à la concentration intrinsèque du GaAs. Pour les valeurs précédentes des durées de vie et une profondeur de jonction égale à $1,30 \mu \mathrm{m}$ le rendement de conversion atteint $21,7 \%$.

L'effet de la profondeur de la jonction et de la durée de vie des porteurs, dans la couche PGaAs, sur le

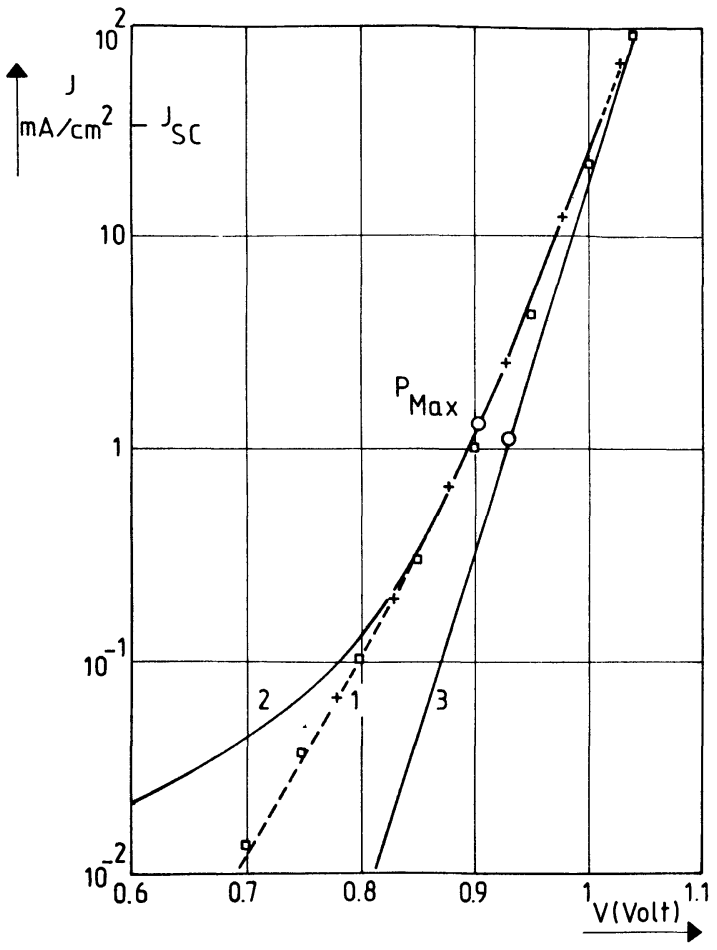

Fig. 3. - Caractéristiques courant-tension de la cellule (c) : 1 : polarisation directe de la structure à l'obscurité ; $2: J-J_{\mathrm{SC}}$ en fonction de la tension de la cellule éclairée dans les conditions AMO; 3 : loi déduite du modèle analytique simple de diffusion.

[Current-voltage characteristics of cell (c) : $1:$ the structure is forward biased in the dark; $2: J-J_{\mathrm{SC}}$ versus potential when the cell is irradiated with AMO solar spectrum; $3:$ simple diffusion law.]

rendement de conversion est décrit par les courbes de la figure 4. L'optimisation de l'épaisseur de la région de type $\mathrm{P}$ correspond à des valeurs comprises entre 1,1 et $1,3 \mu \mathrm{m}$ dans le cas où les longueurs de diffusion des électrons et des trous atteignent respectivement 3 et $2 \mu \mathrm{m}$.

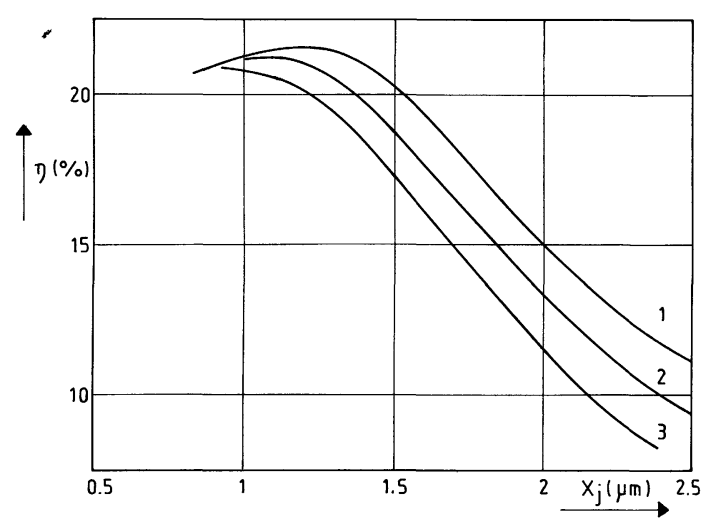

Fig. 4. - Variation du rendement de conversion de la cellule (c) en fonction de la profondeur de jonction et de la durée de vie. Calcul effectué dans les conditions AMO. $\tau_{\mathrm{P}}=10,3 \mathrm{~ns}$ et 1 : $\tau_{\mathrm{N}}=1,4 \mathrm{~ns} ; 2: \tau_{\mathrm{N}}=0,76 \mathrm{~ns} ; 3: \tau_{\mathrm{N}}=0,51 \mathrm{~ns}$.

[Computed conversion efficiency versus junction depth for various lifetime values. Cell (c) under AMO solar spectrum. $\tau_{p}=10.3 \mathrm{~ns}$ and 1: $\tau_{\mathrm{N}}=1.4 \mathrm{~ns} ; 2: \tau_{\mathrm{N}}=0.76 \mathrm{~ns} ; 3: \tau_{\mathrm{N}}=0.51 \mathrm{~ns}$.] 
L'étude précédente est relative à un rayonnement égal à un soleil. Le paragraphe suivant analyse le comportement des cellules solaires sous concentration.

4. Evolution des caractéristiques aux forts éclairements. - 4.1 EFFET DE LA CONCENTRATION SOLAIRE SUR LES PRINCIPAUX PARAMÈTRES PHOTOVOLTAÏQUES. - Un des atouts majeurs du matériau GaAs est son utilisation sous forte concentration de flux lumineux. Dans ce paragraphe la cellule considérée du type $1 b$, définie au paragraphe 2 , est supposée fonctionner à température ambiante $(T=300 \mathrm{~K})$ quel que soit le flux incident.

Admettant la loi de proportionnalité entre la densité de photocourant $J_{\mathrm{L}}$ et l'intensité du rayonnement incident $E_{0}$, le calcul de la tension en circuit ouvert $V_{\text {Co }}$, de la densité de courant de court-circuit $J_{\text {SC }}$ du facteur de forme FF et du rendement de conversion $\eta$, dans le cadre du modèle simplifié, reste bien fondé tant que la concentration des porteurs générés est inférieure à celle des porteurs majoritaires. La figure 5 montre l'évolution des paramètres $\mathrm{FF}$ et $\eta$ de la cellule $1 b$ en fonction du taux de concentration $K$ pour différentes valeurs de la résistance série $R_{\mathrm{S}}$. Nous constatons d'abord que, pour $R_{\mathrm{S}}=0$, le facteur de forme n'est pas rigoureusement constant puisqu'il croît de 0,884 pour $K=1$ à 0,90 pour $K=2000$. Cela signifie que le rendement de conversion $\eta_{K}$ sous $K$ soleils ne suit pas tout à fait une loi linéaire en

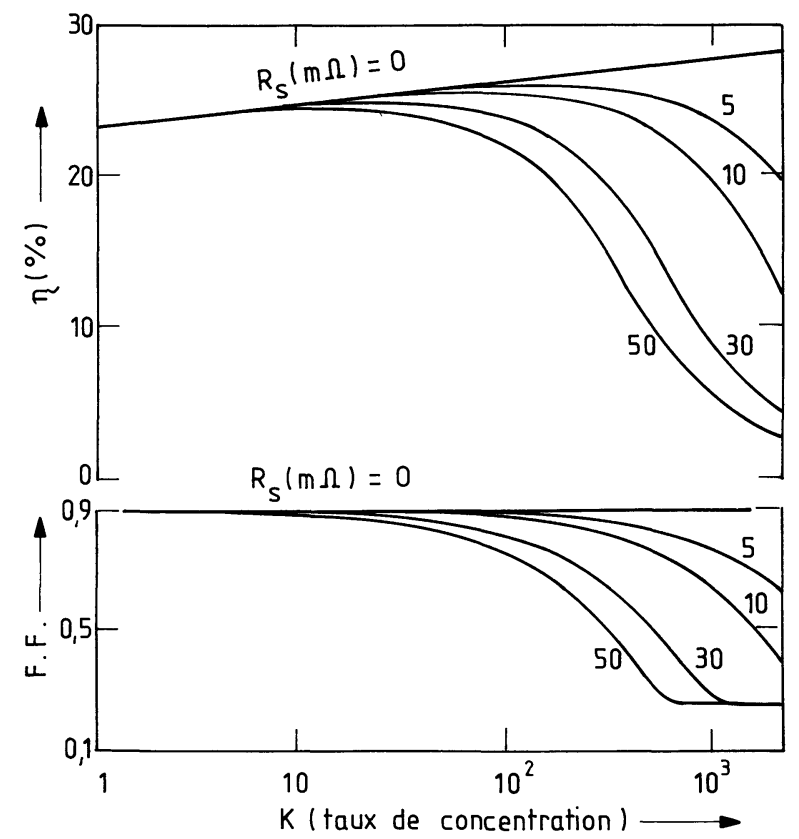

Fig. 5. - Variation du rendement de conversion et du facteur de forme de la cellule (b) en fonction du taux de concentration pour quelques valeurs de la résistance série $R_{\mathrm{S}}$. Calcul effectué à $300 \mathrm{~K}$ dans les conditions AMO.

[Computed conversion efficiency and fill factor at $300 \mathrm{~K}$ versus concentration ratio for a number of series resistance values. Cell (b) under AMO solar spectrum.] échelle semi-logarithmique en fonction de $K$. En effet, d'après les éqs. (15) et (16) du paragraphe 2 , la tension en circuit ouvert calculée sous $K$ soleils s'écrit :

$$
\begin{aligned}
V_{\mathrm{co}} & =\frac{k T}{q} \ln \left(\frac{J_{\mathrm{L}}}{J_{\mathrm{S}}}+1\right) \simeq \frac{k T}{q} \ln \frac{K J_{\mathrm{L} 1}}{J_{\mathrm{s}}} \\
& \simeq V_{\mathrm{CO} 1}+\frac{k T}{q} \ln K
\end{aligned}
$$

$J_{\mathrm{L}}$ se rapportant à $K$ soleils et $V_{\mathrm{CO} 1}, J_{\mathrm{L} 1}$ se rapportant à un soleil.

Utilisant la relation précédente, le rendement $\eta_{K}$ peut donc s'écrire pour $R_{\mathrm{S}}$ nul $\left(J_{\mathrm{SC}} \equiv J_{\mathrm{L}}\right)$ en supposant FF constant :

$$
\begin{aligned}
\eta_{K} & =F F \cdot \frac{V_{\mathrm{CO}} \cdot J_{\mathrm{SC}}}{K E_{0}} \simeq F F \frac{V_{\mathrm{CO} 1} \cdot J_{\mathrm{SC} 1}}{E_{0}}+ \\
& \quad+F F \frac{J_{\mathrm{SC} 1}}{E_{0}} \cdot \frac{k T}{q} \ln K \\
& \simeq \eta_{1} \cdot\left(1+\frac{k T}{q V_{\mathrm{CO} 1}} \cdot \ln K\right)
\end{aligned}
$$

$\eta_{1}$ étant le rendement à un soleil.

La résistance série [12] d'une cellule est définie comme la somme de la résistance de contact $R_{\mathrm{CO}}\left(\Omega \cdot \mathrm{cm}^{2}\right)$, de la résistance de grille métallique et de la résistance de couche superficielle $R_{\mathrm{SH}}(\Omega / \square)$. Cette dernière, dont la contribution est importante à fort niveau d'éclairement, dépend de la résistivité et de l'épaisseur de la couche.

Sur la figure 5, nous observons bien évidemment que plus la résistance est grande, plus la puissance dissipée est importante et plus faible est le taux de concentration donnant le rendement de conversion maximum. Pour cette cellule de surface $1 \mathrm{~cm}^{2}, K$ est trouvé égal à 200 pour $R_{\mathrm{S}}=5 \mathrm{~m} \Omega$ et à 100 pour $R_{\mathrm{S}}=10 \mathrm{~m} \Omega$. Nous remarquons aussi qu'il y a deux points d'inflexion sur les courbes de rendement relatives à $R_{\mathrm{S}}=30 \mathrm{~m} \Omega$ et $R_{\mathrm{S}}=50 \mathrm{~m} \Omega$, ces points étant corrélatifs à un tassement des valeurs des facteurs de forme correspondants, au-delà des taux $K=1000$ et $K=600$ respectivement. En effet, pour des valeurs de $K$ supérieures, les densités de courant de courtcircuit deviennent plus faibles que les densités de photocourant et se saturent même rapidement. Considérant les deux relations (21) et (22), le rendement varie alors comme $V_{\mathrm{co}} / K$, soit décroît en $\ln K / K$.

Enfin nous mentionnons que si la couche superficielle de la cellule considérée plus haut est relativement peu dopée, on peut se retrouver sous fort éclairement dans un régime quasi intrinsèque avec apparition d'une phototension dans la couche à gradient de bande interdite [13], ce qui a pour effet d'augmenter encore le rendement.

4.2 THÉORIE APPROFONDIE. - Le programme numérique détaillé est utilisé pour étudier l'évolution des caractéristiques et des performances des cellules 
(Fig. 1c) en fonction du taux de concentration $K$. Le calcul des courants en fonction du niveau de concentration $K$ et de la tension est tout d'abord effectué pour une résistance série nulle. La température de la cellule est égale à $300 \mathrm{~K}$. Nous observons une linéarité du courant $J_{\mathrm{SC}}$ en fonction de $K$ jusqu'à des taux de 1200 soleils. Les points de puissance maximale en fonction de la concentration sont déduits des caractéristiques courant-tension obtenues de façon précise à partir du programme détaillé. Nous rappelons sur la figure 6 la courbe $-J_{\mathrm{SC}}+J_{\text {total }}$ en fonction de la tension.

Pour des valeurs élevées de l'intensité, la courbe précédente se confond avec la courbe $J(V)$ de la diode polarisée en direct. Nous obtenons une augmentation du facteur d'idéadité de la diode et de son courant de saturation lorsque des densités de courant supérieures à $15 \mathrm{~A} / \mathrm{cm}^{2}$ sont atteintes. Dans ces conditions la densité des charges électriques dans le volume du substrat à proximité de la région de la charge d'espace n'est plus négligeable; il se développe ainsi un champ électrique de sens opposé à celui de la charge d'espace, côté $\mathbf{N}$. La condition de quasi-neutralité n'est plus observée; des phénomènes de haute injection apparaissent.

La caractéristique $J(V)$ nous permet de connaître avec exactitude le courant direct qui traverse la jonction pour chaque valeur de la tension aux bornes de la cellule. Les valeurs du courant de saturation et $\mathrm{du}$ facteur d'idéalité en chaque point de fonctionnement sont ainsi prises en compte dans le calcul du rendement. Une augmentation du taux de concentration ou bien du courant de court-circuit produit, sur la courbe $J(V)$ de la figure 6 , un déplacement du point de puis-

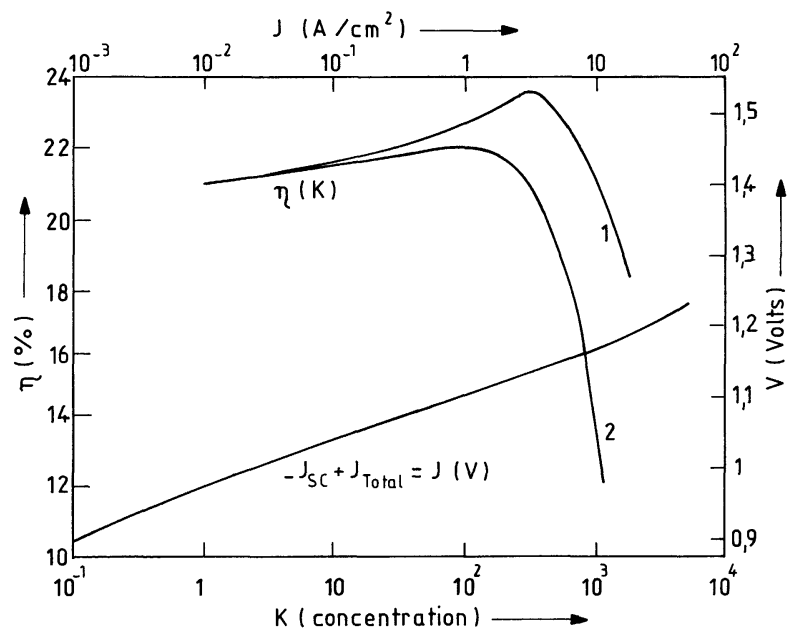

Fig. 6. - Caractéristique théorique courant-tension $J(V)$ et courbe de variation du rendement de conversion $\eta(K)$ de la cellule $(c)$ en fonction du taux de concentration $K: 1:$ pour $R_{\mathrm{S}}=5 \mathrm{~m} \Omega$ et 2 : pour $R_{\mathrm{S}}=15 \mathrm{~m} \Omega$. Calcul effectué à $300 \mathrm{~K}$ dans les conditions AMO.

[Computed $J(V)$ characteristic and conversion efficiency curve $\eta(K)$ versus concentration ratio : $1:$ for $R_{\mathrm{S}}=5 \mathrm{~m} \Omega$ and $2:$ for $R_{\mathrm{S}}=15 \mathrm{~m} \Omega$. Cell (c) at $300 \mathrm{~K}$ under AMO solar spectrum.] sance de sortie maximale, quí ne dépend que des paramètres physiques et technologiques de la cellule. Les valeurs précédentes du courant et de la tension sont les données de l'initialisation à l'itération permettant de déduire la puissance maximale de sortie de la cellule présentant des chutes ohmiques. Les résultats sont portés sous la forme des courbes du rendement en fonction du taux de concentration $K$ pour différentes valeurs de la résistance série (Fig. 6). Les rendements sont encore déterminés pour le spectre solaire AMO. La résistance série qui est décrite dans le paragraphe précédent est déduite à partir d'un modèle de répartition uniforme du courant dans la résistance de couche.

Le calcul précis montre un accroissement de la tension en circuit ouvert, compris entre 30 et $50 \mathrm{mV}$, en fonction du logarithme du taux de concentration. Ces valeurs sont supérieures à la pente de $25 \mathrm{mV}$ par décade déduite des calculs classiques.

Cette variation, encore favorable pour une cellule de $1 \mathrm{~cm}^{2}$ présentant une résistance série de $5 \mathrm{~m} \Omega$, est liée à la plus grande contribution de la composante de diffusion au courant total. Le courant de saturation correspondant à la génération-recombinaison dans la région de charge d'espace n'est plus le terme dominant. Bien qu'une diminution du facteur d'idéalité soit calculée, tout se passe comme si la réduction du courant de saturation produisait l'augmentation de la tension en circuit ouvert lorsque le courant d'obscurité augmente. Ce comportement est observé pour des courants de court-circuit compris entre 0,1 et $3 \mathrm{~A} / \mathrm{cm}^{2}$. Le maximum du rendement (Fig. 6) de la cellule solaire de $1 \mathrm{~cm}^{2}$ avec une résistance série de $5 \mathrm{~m} \Omega$ (espacement des doigts $d=100 \mu \mathrm{m}, R_{\mathrm{SH}}=300 \Omega / \square$ et

$$
R_{\mathrm{CO}}=2 \mathrm{~m} \Omega \cdot \mathrm{cm}^{2} \text { ) }
$$

est atteint pour une concentration de 350 environ. La réduction du rendement est importante pour la cellule qui présente une résistance série égale à $15 \mathrm{~m} \Omega$ $\left(d=300 \mu \mathrm{m}, R_{\mathrm{SH}}=400 \Omega / \square, R_{\mathrm{Co}}=1,5 \mathrm{~m} \Omega . \mathrm{cm}^{2}\right)$. Dans ce cas la dégradation des performances apparaît à partir d'un taux de concentration de l'ordre de 200 .

Les variations du rendement, obtenues à partir d'un modèle de résistance série non répartie, en fonction de la concentration, donnent une décroissance de plus en plus accentuée.

Ce résultat ne correspond pas exactement à l'allure des courbes expérimentales qui montrent une légère saturation de la chute du rendement aux forts éclairements.

5. Autres phénomènes physiques contribuant aux performances des cellules solaires au GaAs. - 5.1. EFFETS DE LA TEMPÉRATURE. - Nous avons dans le paragraphe précédent étudié le comportement des cellules au GaAs sous concentration, à température ambiante. Cette analyse n'est valable que pour des cellules terrestres correctement refroidies car, dans 
l'espace, un flux lumineux important implique une élévation de température. Nous étudions donc ici les effets liés à l'échauffement pour diverses valeurs de l'intensité du rayonnement.

Les paramètres du matériau GaAs les plus sensibles à la température sont la concentration intrinsèque des porteurs, le coefficient d'absorption et la largeur de bande interdite, enfin les paramètres électriques : mobilité et durée de vie des porteurs minoritaires.

Nous avons supposé que la largeur de bande interdite du GaAs suit en fonction de la température, au-delà de $T_{\mathrm{a}}=300 \mathrm{~K}$, une loi de développement limité à l'ordre un, ceci étant confirmé par des mesures précises de piézoréflectivité [14] :

avec

$$
E_{\mathrm{g}}(T)=E_{\mathrm{g}}\left(T_{\mathrm{a}}\right)+\left(T-T_{\mathrm{a}}\right)\left(\frac{\mathrm{d} E_{\mathrm{g}}}{\mathrm{d} T}\right)_{T=T_{\mathrm{a}}}
$$

$$
\left(\frac{\mathrm{d} E_{\mathrm{g}}}{\mathrm{d} T}\right)_{T=T_{\mathrm{a}}}=-4 \times 10^{-4} \mathrm{eV} / \mathrm{K}
$$

tandis que le coefficient d'absorption est relié à la température par $E_{\mathrm{g}}$ selon la relation (7).

Pour la concentration intrinsèque, nous avons adopté la loi :

$$
n_{\mathrm{i}}=5,07 \times 10^{14} T^{3 / 2} \exp \left[-E_{\mathrm{g}}(T) / 2 k T\right] .
$$

Les densités de courant de saturation $J_{\mathrm{SN}}$ et $J_{\mathrm{SP}}$, proportionnelles à $n_{\mathrm{i}}^{2}$ croissent donc très rapidement avec $T$.

A haute température, la mobilité n'est influencée que par les phonons du réseau. Etant donné qu'audelà de la température de Debye, voisine de $420 \mathrm{~K}$, la contribution des phonons optiques, prépondérante par rapport à celle des phonons acoustiques, varie en $T^{-1 / 2}$, nous avons admis que dans la gamme de température étudiée, 300 à $600 \mathrm{~K}$, la mobilité décroît comme $T^{-1 / 2}$. Le coefficient de diffusion, lié à la mobilité par la relation d'Einstein $D=\mu(k T / q)$, croît donc comme $T^{1 / 2}$. La durée de vie $\tau$ est supposée croître suivant la loi $\exp [(T-300) / T]$ ainsi que l'ont montré expérimentalement Vilms et Spicer [15]. Par conséquent la longueur de diffusion $L=(D \tau)^{1 / 2}$ varie en $T^{1 / 4} \exp [(T-300) / 2 T]$, c'est-à-dire croît moins vite que la température.

Le calcul a été appliqué à la cellule précédemment étudiée sous concentration (Fig. $1 b$ ), pour voir les effets combinés de la température et de l'intensité $\mathrm{du}$ rayonnement. Nous avons choisi une faible valeur de la résistance série $R_{\mathrm{S}}=10 \mathrm{~m} \Omega$. La figure 7 montre la variation du rendement obtenue, en fonction de la température entre 300 et $600 \mathrm{~K}$, pour quatre valeurs de la concentration. Bien entendu les rendements décroissent lorsque $T$ augmente, mais nous observons que cette décroissance n'est pas constante, plus faible au-dessous de $400 \mathrm{~K}\left(127^{\circ} \mathrm{C}\right)$, plus forte au-dessus. Ce phénomène est probablement lié au

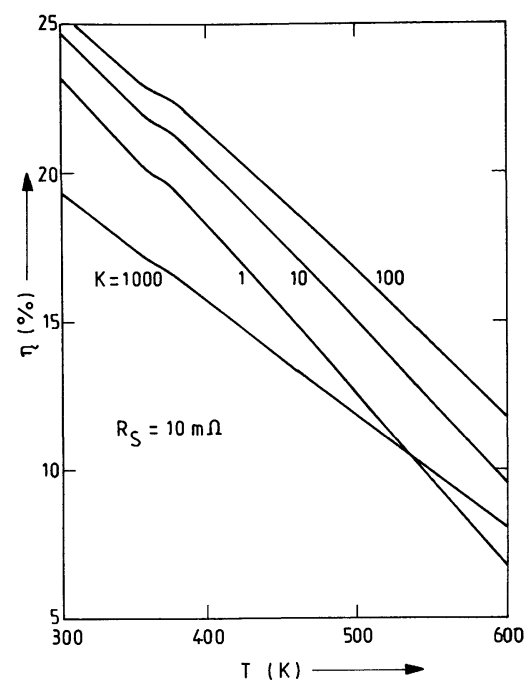

Fig. 7. - Variation du rendement de conversion de la cellule (b) en fonction de la température pour plusieurs valeurs du taux de concentration $K$. Calcul effectué dans les conditions AMO.

[Computed conversion efficiency versus temperature for several concentration ratio values. Cell (b) under AMO solar spectrum.]

déplacement du bord de la bande d'absorption vers les faibles énergies lorsque $T$ augmente, ce qui a un effet important sur l'augmentation du courant de court-circuit. Pour $K=1$, nous trouvons que $\mathrm{d} \eta / \mathrm{d} T$ vaut $0,049 \% .{ }^{\circ} \mathrm{C}^{-1}$ pour $T<400 \mathrm{~K}$ et $0,058 \%{ }^{\circ} \mathrm{C}^{-1}$ pour $T>400 \mathrm{~K}$ alors que Hovel et Woodall [16] ont mesuré des pentes respectives de $0,022 \%{ }^{\circ} \mathrm{C}^{-1}$ jusqu'à $200{ }^{\circ} \mathrm{C}$ et de $0,059 \%{ }^{\circ} \mathrm{C}^{-1}$ au-delà. Plus exactement l'analyse des résultats montre que $V_{\mathrm{CO}}$ décroît presque linéairement avec $T, \mathrm{~d} V_{\mathrm{co}} / \mathrm{d} T$ variant entre 2,02 et $2,11 \mathrm{mV} /{ }^{\circ} \mathrm{C}$ alors que les mêmes auteurs ont déterminé des pentes comprises entre 1,9 et $2,2 \mathrm{mV} /{ }^{\circ} \mathrm{C}$. L'accord est satisfaisant. La croissance de $J_{\mathrm{SC}}$ et la décroissance de $F F$, déterminées théoriquement, sont aussi vérifiées expérimentalement. Les mêmes analyses peuvent être faites pour les autres valeurs de la concentration. Cependant la figure 7 montre bien que la pente $\mathrm{d} \eta / \mathrm{d} T$ décroît avec la concentration de $0,058 \%{ }^{\circ} \mathrm{C}^{-1}$ pour $K=1$ à $0,037 \%{ }^{\circ} \mathrm{C}^{-1}$ pour $K=1000$, résultat analogue à celui trouvé par Hovel [17]. Or, nous avons montré dans le paragraphe 4.1 (voir Fig. 5) que, à la température de $300 \mathrm{~K}$, le rendement de conversion pour $K=1000$ est inférieur à celui trouvé pour $K=1$, à cause des pertes dues à la résistance série $R_{\mathrm{S}}=10 \mathrm{~m} \Omega$. Ces deux remarques permettent d'expliquer le croisement des courbes sur la figure 7. Enfin le résultat global de cette figure est que l'intérêt des cellules solaires au GaAs sous concentration n'est manifeste que si, d'une part on minimise la résistance série par l'augmentation du nombre de doigts dans la grille collectrice, d'autre part on arrive à évacuer suffisamment la chaleur à l'aide d'un radiateur ou d'un liquide réfrigérant. 
5.2 EFFET DE LA RÉPARTITION NON UNIFORME DE LA DENSITÉ DE COURANT DANS LA COUCHE ÉPITAXIÉE. - La cellule solaire illuminée par un flux solaire concentré est le siège d'un courant de court-circuit de plusieurs ampères par unité de surface $\left(\mathrm{cm}^{2}\right)$. Une partie du courant généré traverse latéralement [18] la couche épitaxiée, résistive, avant d'atteindre la région métallisée où se produit la collecte. La répartition dú potentiel dans le plan parallèle à la surface illuminée n'est plus homogène. La chute de tension dans la couche P-GaAs donne une polarisation directe de la jonction P-N plus prononcée pour la région la plus éloignée du métal. Il en résulte que, pour le fonctionnement normal de la cellule solaire, la densité du courant total est plus élevée près du contact où le courant d'obscurité est le plus faible. La proximité du contact collecteur est le siège de l'effet de focalisation des lignes de courant ; ce phénomène est l'équivalent de la défocalisation observée dans la base des transistors bipolaires.

La section de la cellule la plus éloignée des doigts métalliques, là où la polarisation de la jonction est la plus grande, devient de plus en plus inactive à la contribution du courant total. Etant donné un espacement interdoigts, une augmentation du taux de concentration produit une extension de la région inactive de la cellule. La diminution de l'intensité dans la couche épitaxiée, suivant le plan parallèle à la jonction, atténue l'effet de la chute ohmique due à la résistance carrée, de couche. La contribution de la région superficielle, de type $\mathrm{P}$, à la résistance série est réduite à la région juxtaposée aux doigts métalliques; cette partie est pratiquement traversée par le courant suivant la direction perpendiculaire à la jonction.

La chute de potentiel due à la résistance de couche $R_{\mathrm{SH}}(\Omega / \square)$ lors du passage du courant de court-circuit $J_{\mathrm{SC}}$ dans une cellule présentant un espacement entre les doigts égal à $d$, est approchée par la relation suivante :

$$
\Delta V=\frac{R_{\mathrm{SH}} \cdot J_{\mathrm{SC}} \cdot d^{2}}{12} .
$$

Ainsi pour une cellule. solaire dont la résistance carrée est égale à $200 \Omega / \square$, une distance $d$ de $250 \mu \mathrm{m}$, un taux de concentration de 100 soleils

$$
\left(J_{\mathrm{SC}} \simeq 2,7 \mathrm{~A} / \mathrm{cm}^{2}\right)
$$

produit une chute de tension de l'ordre du potentiel thermodynamique, $U_{\mathrm{T}} \simeq 26 \mathrm{mV}$.

La réduction du rendement est importante sous 200 soleils (Fig. 8, réf. [19]).

La saturation de la chute du rendement est observée pour un $J_{\mathrm{SC}}$ de l'ordre de $5 \mathrm{~A} / \mathrm{cm}^{2}$.

\subsection{CAS DES CELlules COMPORTANT DES CONCEN-} TRATIONS ÉLEVÉES D'IMPURETÉS. - La densité d'états au bas de la bande de conduction est très inférieure à la densité d'états au haut de la bande de valence du GaAs :

$N_{\mathrm{c}}=4,4\left(10^{17}\right) \mathrm{cm}^{-3}$ et $N_{\mathrm{v}}=1,51\left(10^{19}\right) \mathrm{cm}^{-3}$.

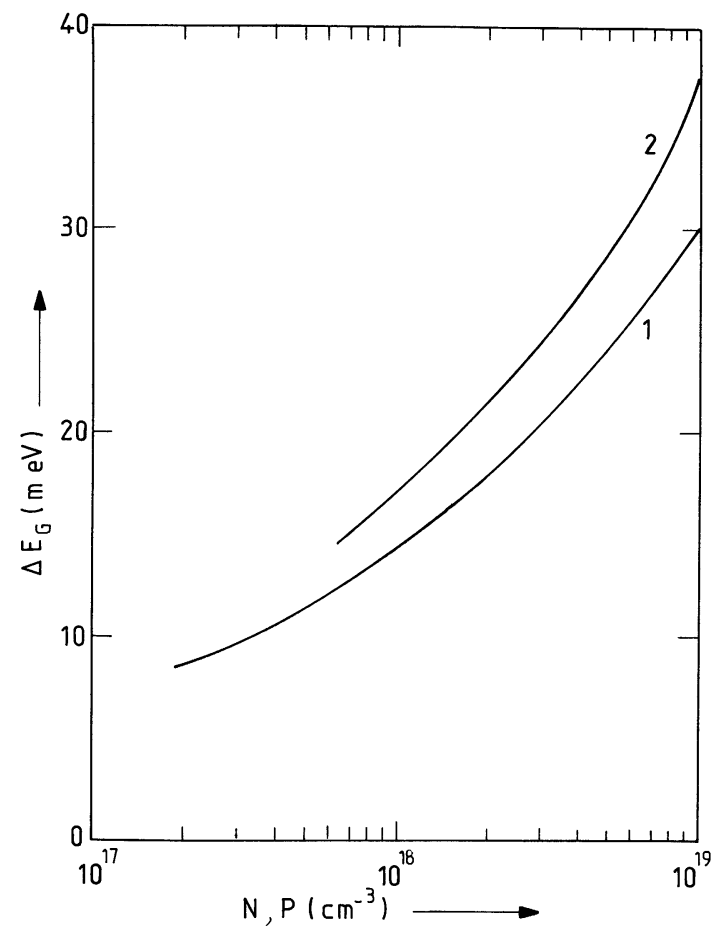

Fig. 8. - Réduction de la bande interdite du GaAs en fonction du nombre total des électrons $\mathbf{N}$ et des trous $\mathbf{P}: 1$ : suivant la relation (26) et 2 : d'après les études de L. P. Zverev [21].

[GaAs bandgap reduction versus the whole electrons $\mathrm{N}$ and holes $\mathrm{P}$ densities : 1 : according to the result (26) and $2:$ after L. P. Zverev's work [21].]

L'expression des porteurs suivant les lois déduites de la statistique de Maxwell-Boltzmann ne peut plus être retenue pour l'optimisation des structures $\mathrm{N}-\mathrm{P}$ comportant une densité d'impuretés donatrices supérieure à quelque $10^{17} \mathrm{~cm}^{-3}$. Le calcul des distributions en énergie des porteurs suivant la statistique de FermiDirac donne une densité des électrons inférieure à la valeur déduite par la statistique de Maxwell-Boltzmann [20]. Parallèlement à la dégénérescence du semiconducteur fortement dopé, les phénomènes de réduction de la bande interdite apparaissent.

Deux types d'interaction sont responsables de la variation de la largeur de la bande interdite. Un premier mécanisme physique est l'extension en énergie des niveaux des impuretés qui formeront l'équivalent d'une bande dont la limite extérieure se rapproche $\mathrm{du}$ bas de la bande de conduction du GaAs de type $\mathrm{N}$ par exemple. Un deuxième mécanisme est le déplacement des limites des bandes de conduction et de valence lié aux phénomènes d'interaction des porteurs libres [21]. Une réduction de l'ordre de $20 \mathrm{meV}$ est observée pour l'effet associé à une densité de $10^{18} \mathrm{~cm}^{-3}$ des atomes donneurs du N-GaAs. Le second mécanisme lié à la perturbation due à l'énergie d'échange entre les porteurs libres est beaucoup plus important. Les courbes de la figure 8 rappellent la réduction $\Delta E_{\mathrm{g}}$ (meV) en fonction de la densité des porteurs libres. Nous observons une variation de $25 \mathrm{meV}$ pour 
$N=2\left(10^{18}\right) \mathrm{cm}^{-3}$ et de plus de $35 \mathrm{meV}$ pour $P=7\left(10^{18}\right) \mathrm{cm}^{-3}$, (courbe 2). Nous portons sur la figure précédente la variation donnée par la loi [22] représentative de la diminution de la bande interdite (courbe 1) :

$$
\Delta E_{\mathrm{g}}=-1,42 \times 10^{-8}\left(P^{1 / 3}+N^{1 / 3}\right)(\mathrm{eV})
$$

où $N$ et $P$ représentent les densités totales des électrons et des trous respectivement. Le résultat (26) reste inférieur aux valeurs expérimentales obtenues dans les travaux publiés dans la référence [21]. D'autres travaux [23] ont par contre mis en évidence théoriquement des variations de largeur de bande supérieures.

Les phénomènes précédents doivent être pris en compte dans le calcul des performances des cellules solaires. La variation $\Delta E_{\mathrm{G}}$ de la largeur de la bande interdite produit une composante supplémentaire du champ électrique agissant sur les porteurs minoritaires. Les effets relatifs aux dopages élevés tendent à diminuer la durée de vie des porteurs et contribuent à une dégradation du taux de collecte.

6. Conclusion. - Nous avons décrit à l'aide de plusieurs modèles le comportement des cellules solaires au GaAlAs/GaAs avec ou sans gradient de bande interdite dans la couche superficielle. Un modèle simplifié permet par une étude comparative de choisir la cellule ayant le meilleur rendement. Une étude approfondie prenant en compte les mécanismes de génération-recombinaison dans la zone de transition de la jonction met en évidence que le modèle précédent surestime la valeur du rendement de conversion. Les caractéristiques simulées, tracées en échelle semilogarithmique, font apparaître une non-linéarité des lois de conduction lorsque les durées de vie sont de l'ordre de $1 \mathrm{~ns}$ et $10 \mathrm{~ns}$ respectivement pour les électrons et les trous.

Ce travail montre clairement les effets de la température sur le rendement de conversion des cellules au GaAs, que ce soit avec ou sans concentration et met en évidence son changement de pente $\mathrm{d} \eta / \mathrm{d} T$ autour de $130^{\circ} \mathrm{C}$, observé expérimentalement. Les effets dus à la résistance série sont analysés aux forts niveaux de courant. Les résultats de cette analyse laissent entrevoir que sans trop perdre sur le rendement, des concentrations de 400 sont possibles avec ces cellules de surface $1 \mathrm{~cm}^{2}$ si la résistance série des structures concernées ne dépasse pas $10 \mathrm{~m} \Omega$.

Enfin l'incidence des fortes concentrations d'impuretés, caractérisée notamment par une diminution de la largeur de la bande interdite est analysée quant à ses effets sur le rendement de collecte.

Remerciements. - Les auteurs remercient Monsieur H. Martinot pour l'extension du programme numérique aux structures à gradient de bande interdite et également Monsieur Y. Marfaing pour sa contribution à la correction du manuscrit.

\section{Bibliographie}

[1] Woodall, J. M. and Hovel, H. J., Appl. Phys. Lett. 30 (1977) 492.

[2] Sahai, R., Edwall, D. D. and Harris, J. S. Jr., Appl. Phys. Lett. 34 (1979) 147.

[3] Davis, R. and Knight, J. R., Sol. Energy 17 (1975) 145.

[4] James, L. W. and Moon, R. L., Conf. Rec. 11th IEEE Photovoltaic Specialists Conf., Scottsdale, AZ, May 1975, 402.

[5] Kamath, G. S., Ewan, J. and Knechtli, R. C., IEEE Trans. Electron Devices ED 24 (1977) 473

[6] Fabre, E., Briere, A. and André, J. P., Proc. Int. Conf., 1st E.C. Photovoltaic Solar Energy Conference, Luxembourg, 1977, Reidel Publishing Co., 388.

[7] Fan, J. C. C. and Bozler, C. O., Conf. Rec. 13th IEEE Photovoltaic Specialists Conf., Washington, D.C., June 1978, 953.

[8] Thekaekara, M. P., Sol. Energy 18 (1976) 309.

[9] Bourée, J. E., Revue Phys. Appl. 12 (1977) 559.

[10] Heydemann, M., Thèse de Docteur-Ingénieur, Université Paris-Sud, 1972, Résolution numérique des équations bidimensionnelles de transport dans les semiconducteurs.

[11] Therez, F., Martinot, H. et Esteve, D., Proc. Int. Conf., 2nd E.C. Photovoltaic Solar Energy Conference, Berlin (West), 1979, Reidel Publishing Co., 926.
[12] Handy, R. J., Solid-State Electron. 10 (1967) 765.

[13] Marfaing, Y., Proc. Int. Coll. Solar Cells (Gordon and Breach) $1970,67$.

[14] Camassel, J., Auvergne, D. and Mathieu, H., J. Appl. Phys. 46 (1975) 2683.

[15] Vilms, J. and Spicer, W. E., J. Appl. Phys. 36 (1965) 2815.

[16] Hovel, H. J. and Woodall, J. M., J. Electrochem. Soc. 120 (1973) 1246.

[17] Hovel, H. J., IBM J. Res. Dev. 22 (1978) 112

[18] Therez, F., Solid State Electron. 20 (1977) 941.

[19] Sahai, R., Edwall, D. D. and Harris, J. S. Jr., Conf. Rec. 13th IEEE Photovoltaic Specialists Conf., Washington, D.C., June 1978, 946.

[20] Shibib, M. A., Lindholm, F. A. and Therez, F., IEEE Trans. Electron Devices ED-26 (1979) 959.

[21] Zverev, L. P. et al., Sov. Phys. Semicond. 11 (1977) 603.

[22] Kressel, H. and Butler, J. K., Semiconductor Lasers and Heterojunction LEDs (Academic Press) 1977, 36.

[23] Inkson, J. C., J. Phys. C 9 (1976) 1177. 